\title{
Magnitude, padrão e gravidade da multimorbilidade em idosos assistidos pelas equipas de Cuidados Continuados Integrados: estudo transversal
}

Paula Maria Broeiro-Gonçalves, ${ }^{1}$ Pedro Aguiar, ${ }^{2}$ Isabel Loureiro ${ }^{3}$

\section{RESUMO}

Objetivos: Caracterizar a magnitude, gravidade e padrão da multimorbilidade e verificar que associação existe entre as dimensões de multimorbilidade e condições médicas ou sistemas.

Tipo de estudo: Estudo transversal, descritivo com componente analítica, por entrevista.

Local: Equipas de Cuidados Continuados Integrados (ECCI) da região de Lisboa e Vale do Tejo.

População: Idosos com 75 e mais anos.

Métodos: Calculou-se a dimensão da amostra ( $n=114$ idosos) para uma margem de erro de $5 \%$ e um intervalo de confiança de $95 \%$, corrigida para o efeito de desenho ( $n=228$ idosos). A amostragem foi efetuada por clusters (ECCI) aleatoriamente selecionados. A análise foi realizada através do modelo linear generalizado - GEE com recurso à ferramenta IBM SPSS, versão 24.0 para o sistema operativo MAC Os.

Resultados: Participaram 230 idosos, 54\% do sexo feminino, com média de idade de 83,6 anos, pouco escolarizados (40\% sem escolaridade) e $14,8 \%$ a residir sós. O total de diferentes condições médicas reportadas foi de 121 . O número médio de problemas por pessoa foi de 9,5 e o índice de Charlson médio de 8,5. Verificou-se associação ao sexo masculino tanto para a magnitude $(O R=2,452)$ como para gravidade $(O R=22,333)$ da multimorbilidade. Estiveram associadas a gravidade: a multimorbilidade definida como seis ou mais condições $(O R=22,333)$ e três ou mais sistemas $(O R=3,171)$. A coronariopatia isquémica foi o diagnóstico associado ao maior: número médio de condições $(11,64)$ e índice de Charlson $(10,50)$. A hipertensão e a insuficiência cardíaca foram os diagnósticos com mais problemas associados e a mais que três sistemas.

Conclusões: A população em ECCl é idosa e com elevada multimorbilidade. Confirmou-se a diferença entre sexos com as mulheres mais velhas e os homens com maior multimorbilidade e mais grave. A definição de multimorbilidade de seis ou mais problemas esteve associada a doença multissistémica e a gravidade.

Palavras-chave: Multimorbilidade; Comorbilidade; Padrão de multimorbilidade; Idosos; Visita domiciliária.

\section{INTRODUÇÃO}

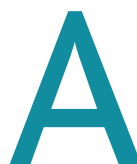
multimorbilidade, enquanto coexistência de mais que uma condição médica numa pessoa, em idade avançada, não tem reunido consenso quanto à definição e ao modo como tem sido estudada. ${ }^{1-4}$ De forma consistente, a multimorbilidade tem sido associada à população de mais idade, ao sexo feminino e ao baixo nível socioeconómico; ${ }^{5}$ porém, o estudo português de Prazeres e cola- boradores veio contrariar a associação ao sexo feminino. ${ }^{6}$

Originalmente, multimorbilidade foi definida como duas ou mais doenças. No entanto, tem sido debatido se

1. UCSP dos Olivais, ACeS Lisboa Central. Faculdade de Medicina de Lisboa. INFARMED, Comissão de Avaliação de Medicamentos. ORCID 0000-0002-5013-6171 2. Professor Auxiliar de Epidemiologia e Estatística. Escola Nacional de Saúde Pública, Universidade Nova de Lisboa. ORCID 0000-0002-0074-7732

3. Professora Catedrática de Promoção de Saúde. Escola Nacional de Saúde Pública, Universidade Nova de Lisboa. ORCID 0000-0001-7374-6258 
três ou mais doenças não seria uma melhor medida, em particular, nas pessoas de mais idade. ${ }^{3,7}$ Apesar de uma estimativa de prevalência mais baixa, facilitaria a identificação de pacientes com maiores necessidades de saúde e, consequentemente, seria mais útil para os médicos. ${ }^{7}$

A multimorbilidade é mais do que a simples agregação de patologias individuais e tem implicações na gestão de doença, ${ }^{8}$ está associada ao aumento da utilização dos serviços e a piores resultados em saúde. ${ }^{8-11}$ É, pois, necessário, estudá-la nas suas diferentes dimensões: magnitude (e.g., contagem de diagnósticos), gravidade (e.g., índice de Charlson) ${ }^{12}$ ou padrão (e.g., agregados de diagnósticos) $)^{2,13}$.

A contagem de doenças definida como uma simples enumeração não ponderada do número de doenças por pessoa tem sido a medida mais comummente estudada. ${ }^{13} \mathrm{~A}$ compreensão do padrão de multimorbilidade pode facilitar o diagnóstico, a integração de cuidados e melhorar a qualidade de vida dos pacientes. ${ }^{8-9,14}$ É reconhecida a relevância de se compreender o padrão de combinações ou agregados de doenças crónicas; no entanto, a sua investigação permanece limitada. ${ }^{8}$ Existe, pois, uma necessidade crescente de conhecer os padrões de morbilidade e a complexidade dos agregados. ${ }^{9}$ Os padrões ou agregados de multimorbilidade podem indicar associações de doenças por frequência, sem uma explicação causal, ${ }^{14}$ ou por agregação causal que agrupa doenças com relação fisiopatológica entre si. ${ }^{10,14}$

A noção de gravidade, definida pelo peso total de disfunção fisiológica ou pelo peso total de doenças com impacto sobre um indivíduo, ${ }^{15}$ outra das dimensões da multimorbilidade, tem sido transmitida pelo termo comorbilidade e medida através de índices. O índice de Charlson é um dos mais amplamente utilizados para medir gravidade de doença, utilizando morbilidades com diferente impacto no seu risco relativo de morte. ${ }^{12} \mathrm{O}$ índice global ou índice de Charlson permite aferir a presença de multimorbilidade (e.g., índice de 3 ou mais pontos significa alta multimorbilidade) e predizer a mortalidade (e.g., índice de 5 ou mais pontos é preditor de morte a três anos em $85 \%$ dos doentes). ${ }^{12,16}$ O índice de Charlson, ${ }^{12}$ embora tenha sido desenvolvido e validado em pacientes hospitalizados, foi adaptado e validado em cuidados primários e populações comunitárias. ${ }^{13}$

A complexidade da gestão da multimorbilidade, da fragilidade ou da dependência trouxe novos desafios aos sistemas de saúde. ${ }^{9,17}$ A par destas mudanças, a maioria das pessoas de mais idade (75 e mais anos) preferia ser cuidada e morrer em casa, se lhes fosse permitido escolher. ${ }^{18}$ Portugal tem-se adaptado às mudanças sociodemográficas e de necessidades sociais e de saúde, criando e mantendo, desde 2006, a Rede Nacional de Cuidados Continuados (RNCCI) no âmbito dos Ministérios da Saúde e do Trabalho e da Solidariedade Social. ${ }^{19-21}$ A prestação de cuidados de saúde e de apoio social é assegurada por diferentes tipologias de cuidados que incluem as equipas domiciliárias [Equipas de Cuidados Continuados Integrados (ECCI)], ${ }^{22}$ assumindo-se que os doentes que recebem cuidados no domicílio pelas ECCI não têm critérios de gravidade que requeiram internamento hospitalar e têm suporte familiar e social que o permita. ${ }^{21-22}$

Em 2016, a população da RNCCI com idade superior a 65 anos representou $81,6 \%$, o sexo feminino $54,2 \%$ do total de utentes, em que $29,9 \%$ das mulheres tinha idade superior a 80 anos. O perfil sociodemográfico dos utilizadores da RNCCI reflete o perfil de envelhecimento da população portuguesa. ${ }^{23}$

Uma vez que os doentes em ECCI não reúnem critérios de internamento hospitalar, em idosos ao cuidado das ECCI da região de Lisboa e Vale do Tejo definiu-se como objetivo primário do estudo caracterizar a magnitude, gravidade e padrão da multimorbilidade. Outro objetivo foi verificar que associação existe entre condições médicas e/ou aparelhos e sistemas e as dimensões de multimorbilidade (magnitude e gravidade).

\section{MÉTODOS}

Desenhou-se um estudo transversal, descritivo com componente analítica, por entrevista. Por uma questão de exequibilidade definiu-se, como área de abrangência, a região de Lisboa e Vale do Tejo e planeou-se entrevistar os cuidadores dos doentes idosos assistidos pelas ECCI.

Calculou-se a dimensão da amostra ( $n=114$ idosos) tendo em conta a população ( $N=324.878$, idosos com 75 e mais anos da região de Lisboa e Vale do Tejo), ${ }^{24}$ a prevalência de multimorbilidade neste grupo etário (92\%), ${ }^{6}$ uma margem de erro de $5 \%$ e um intervalo de confiança de 95\% (IC95).

Atendendo a que não era possível a aleatorização dos participantes por se encontrarem apenas tempo- 
rariamente ao cuidado das ECCI planeou-se uma amostragem por clusters. Tratando-se de uma amostragem por clusters corrigiu-se a dimensão da amostra para um eventual efeito do desenho, assumindo-o como igual a 2 ( $n=228$ idosos), como considerado adequado em estudos epidemiológicos. ${ }^{25}$

Selecionou-se uma amostra representativa tendo em conta o processo de amostragem por cluster, à semelhança de outros estudos epidemiológicos. ${ }^{25-29} \mathrm{O}$ processo de amostragem foi realizado em duas etapas:

- A primeira por aleatorização simples das ECCI (clusters) de forma a garantir a dimensão amostral. Esta etapa esteve dependente da capacidade instalada nesta tipologia de cuidados - 2.066 «lugares» distribuídos por 59 ECCI, na ARSLVT, da taxa de ocupação média, da taxa de resposta e das perdas previsíveis por incumprimento dos critérios de elegibilidade.

- A segunda consistiu em convidar todos os participantes que cumprissem os critérios de elegibilidade, no período programado para a colheita de dados em cada ECCI.

Definiram-se como critérios de elegibilidade:

> De inclusão:

- Doente a receber cuidados da ECCI;

- Doente com idade igual ou superior a 75 anos;

- Doente que tivesse cuidador informal (não remunerado);

- Doente que aceitasse participar e desse consentimento informado.

> De exclusão:

- Doente institucionalizado à data da entrevista;

- Doente com idade inferior a 75 anos ou

- Ambos, cuidador e doente, não soubessem ler nem escrever.

As variáveis estudadas foram as sociodemográficas e as de morbilidade (utilizou-se a escala de Charlson ${ }^{16}$-índice e cada um dos problemas dicotomizados, aos quais se acrescentaram os problemas de saúde mais frequentes na população idosa e os diagnósticos a reportar pelo cuidador, à semelhança do estudo australiano ${ }^{3}$ no item «Outros»). Tanto para a questão aberta como para os dados reportados de morbilidade, a confirmação diagnóstica foi feita através da análise da terapêutica em curso e da consulta da informação clínica disponível (e.g., notas de alta hospitalar ou relatórios clínicos).
A unidade de observação foi o binómio cuidador/ /doente através de entrevista ao cuidador informal. Definiu-se como cuidador informal qualquer pessoa que prestasse cuidados gratuitamente por ter uma relação afetiva (e.g., amigos ou vizinhos) ou de parentesco.

A justificação para a avaliação de o doente ser reportada pelo cuidador prendeu-se com a intenção de não excluir do estudo as patologias do doente com repercussão nas suas capacidades cognitivas (e.g., demência).

A colheita de dados ocorreu entre dezembro de 2015 e julho de 2016, no domicílio dos doentes, por aplicação de um questionário sob formato de entrevista realizada pelo investigador ao cuidador, durante a visita da equipa (ECCI) ou em visita programada com o cuidador. O questionário aplicado foi construído tendo em conta as variáveis que se pretendiam estudar após ter sido realizado pré-teste de legibilidade, compreensibilidade e aplicabilidade em 12 pessoas.

A recolha de dados foi padronizada por aplicação do mesmo instrumento a todos os participantes pelo investigador, isto é, foram sempre entrevistados os cuidadores na presença do idoso, o qual, quando cognitivamente bem, colaborava na entrevista.

Durante a entrevista os dados foram registados num ficheiro word, em seguida exportados automaticamente para uma matriz excel e, posteriormente, para a ferramenta estatística IBM SPSS, v. 24.0 para o sistema operativo MAC Os.

No presente estudo, a magnitude da multimorbilidade foi realizada através da soma do número de condições médicas na mesma pessoa. Atendendo à elevada prevalência de multimorbilidade abaixo de seis condições médicas por pessoa, aplicando o modelo de análise de equações de estimação generalizada (GEE) verificou-se a ausência de casos suficientes para cruzamento, sendo, por isso, este o ponto de corte (MM6+) considerado para análise da magnitude.

No índice de Charlson a máxima gravidade, sem ajuste para a idade, foi conferida pelo valor $\geq 5$ : ponderando a idade somou-se o valor 4, correspondendo ao valor atribuído ao grupo etário $\geq 75$ anos. ${ }^{12,30-31}$ Justifica-se, assim, o ponto de corte do índice de Charlson $\geq 9$ para a gravidade da multimorbilidade.

A análise dos dados teve duas componentes, uma descritiva e outra analítica. Considerando o desenho de 


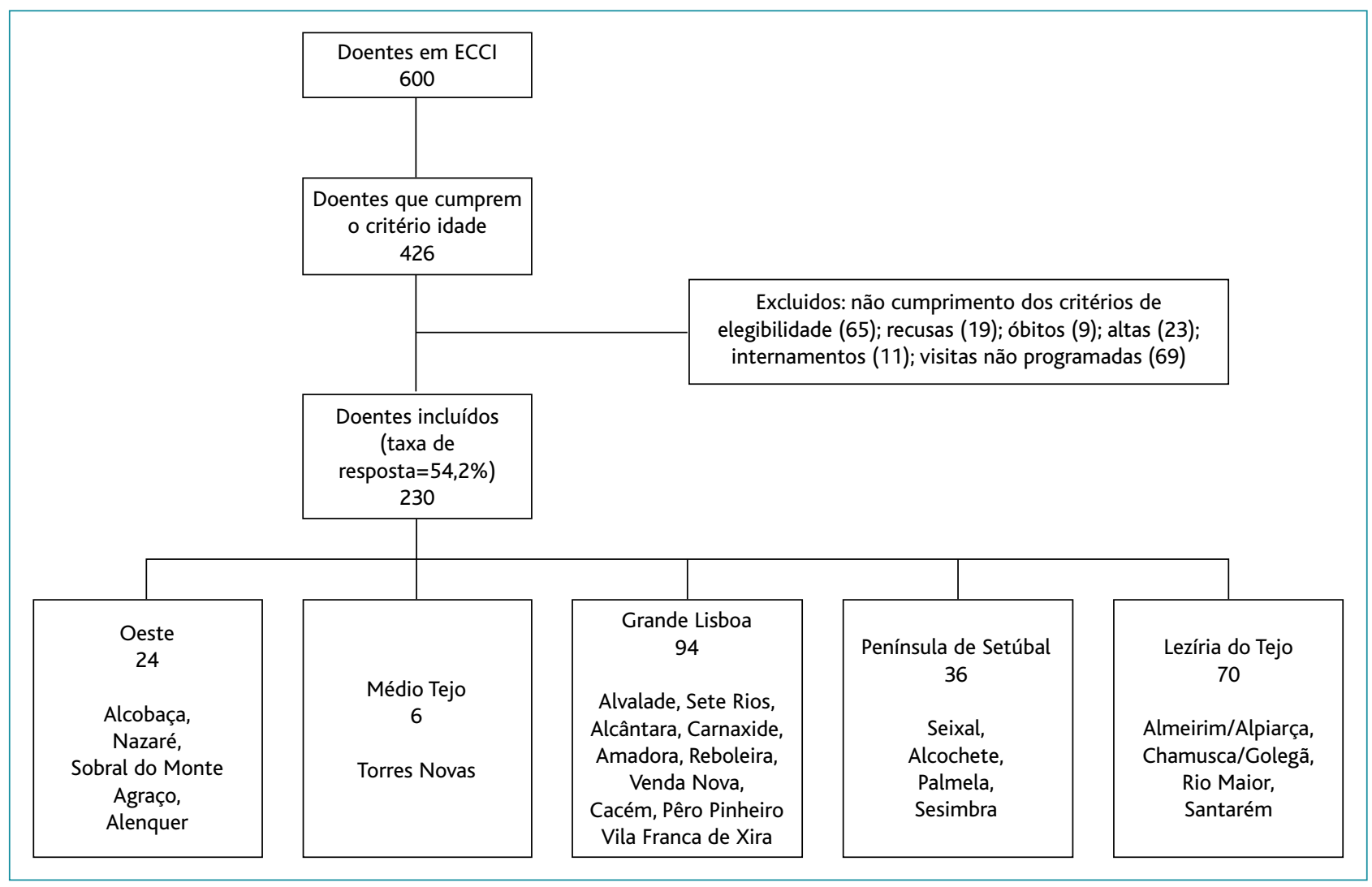

Figura 1. Fluxograma de cumprimento dos critérios de elegibilidade dos participantes e sua distribuição por regiões (NUTS III).

estudo e a amostragem, na análise estatística utilizou-se o modelo de regressão linear generalizado (GLM) através do modelo GEE. Na análise bivariável o modelo GEE foi construído tendo em conta a variável dependente, com distribuição binominal, utilizando a função logit para o cluster ECCI.

$\mathrm{Na}$ análise multivariável considerou-se incluir no modelo, como covariáveis, cada diagnóstico que correspondesse a uma frequência superior a $25 \%$ para cada uma das categorias em estudo: multimorbilidade (MM6+), gravidade (ponto de corte $\geq 9$ do índice de Charlson). Considerou-se, como outras covariáveis a incluir no modelo, as sociodemográficas (idade, sexo, isolamento e escolaridade). O isolamento social foi considerado como covariável sociodemográfica pela sua frequência no grupo etário em estudo. ${ }^{23}$

\section{Requisitos éticos}

No estudo cumpriram-se requisitos éticos como o respeito pela autonomia dos participantes e a confi- dencialidade dos dados.

O estudo obteve parecer favorável da Comissão de Ética para a Saúde da ARSLVT e da Comissão Nacional de Proteção de Dados.

\section{RESULTADOS}

No seguimento do planeamento do estudo procedeu-se à análise descritiva dos resultados, à qual se seguiu a inferencial em duas etapas (bivariável e multivariável).

O fluxograma (Figura 1) representa as perdas de participantes, incluindo a justificação.

Dos 230 participantes, $54 \%$ eram do sexo feminino, a média de idades de 83,6 anos, sendo as mulheres mais velhas e com menor magnitude (número médio de problemas) e menor gravidade (média do índice de Charlson) de multimorbilidade que os homens. O Quadro I apresenta a descrição da amostra estudada.

Relativamente aos aspetos sociodemográficos trata-se de uma população pouco escolarizada. Também a 


\begin{tabular}{|c|c|c|}
\hline \multicolumn{3}{|c|}{$\begin{array}{l}\text { QUADRO I. Distribuição dos idosos por regiōes, suas característica } \\
\text { sociodemográficas e relação de afinidade com o cuidador }\end{array}$} \\
\hline \multirow{6}{*}{$\begin{array}{l}\text { Região (NUTS III) } \\
\text { Frequência (\%) }\end{array}$} & & \\
\hline & Oeste & $24(10,4)$ \\
\hline & Médio Tejo & $6(2,6)$ \\
\hline & Grande Lisboa & $94(40,9)$ \\
\hline & Península de Setúbal & $36(15,7)$ \\
\hline & Lezíria do Tejo & $70(30,4)$ \\
\hline $\begin{array}{l}\text { Idade } \\
\text { Média em anos }\end{array}$ & \multicolumn{2}{|l|}{ 83,6 [F: 84,9 M: 82,1] } \\
\hline \multirow{4}{*}{$\begin{array}{l}\text { Grupos etários } \\
\text { Frequência (\%) }\end{array}$} & 45 a 79 anos & $67(29,1)$ \\
\hline & 80 a 84 anos & $67(29,1)$ \\
\hline & 85 a 89 anos & $55(23,9)$ \\
\hline & 90 a 99 anos & $41(12,2)$ \\
\hline $\begin{array}{l}\text { Sexo } \\
\text { Frequência (\%) }\end{array}$ & \multicolumn{2}{|l|}{ F $124(54,0) ;$ M $106(46,0)$} \\
\hline \multirow{4}{*}{$\begin{array}{l}\text { Escolaridade } \\
\text { Frequência (\%) }\end{array}$} & Sem escolaridade & $93(40,4)$ \\
\hline & 1. ${ }^{\circ}$ Ciclo de escolaridade & $111(48,3)$ \\
\hline & 2. ${ }^{\circ}$ Ciclo ao Secundário & $22(9,6)$ \\
\hline & Formação superior & $4(1,7)$ \\
\hline \multirow{5}{*}{$\begin{array}{l}\text { Relação de parentesco } \\
\text { Frequência (\%) }\end{array}$} & \multicolumn{2}{|l|}{$296(98,0)$} \\
\hline & Esposa(o) & $108(47,0)$ \\
\hline & Filha(o) & $88(38,9)$ \\
\hline & Nora/Genro & $13(5,7)$ \\
\hline & Neta(o) & $8(3,5)$ \\
\hline $\begin{array}{l}\text { Multimorbilidade } \\
\text { Média }\end{array}$ & \multicolumn{2}{|c|}{$\begin{array}{l}\text { N. }{ }^{\circ} \text { de condições por pessoa 9,5 [F:9,02 M:10,0] } \\
\text { Índice de Charlson 8,5 [F:7,96 M: 9,1] }\end{array}$} \\
\hline
\end{tabular}

O número de condições médicas por pessoa permitiu-nos calcular o número médio de problemas por grupo etário e sexo (Figura 2). O número médio de problemas ou condições médicas por pessoa foi de 9,5 . Verifica-se que em todos os grupos etários o número médio de condições médicas foi superior no sexo masculino.

Observando as curvas do número médio de problemas verifica-se um comportamento diferente entre sexos. Na curva relativa ao sexo masculino existe um pico aos 85/89 anos e uma curva ascendente no grupo mais idoso. No sexo feminino existe um esboço de um pico aos 80/84 anos, seguindo-se um decréscimo progressivo até à idade mais avançada. Através do modelo GEE confirmou-se a associação estatisticamente significativa entre o número de problemas e o sexo masculino (OR=2,452; IC95\% 1,341-4,485; $p=0,004)$; contudo, não se verificou associação estatisticamente significativa com a idade.

Relativamente à idade, como o estudo foi realizado com idosos não se encontrou diferença entre grupos etários. Contudo, a curva da Figura 2 sugere haver um declínio no número médio de condições médicas no grupo dos mais idosos (90 e mais anos).

Para o estudo da multimorbilidade na presente amostra de grandes idosos utilizou-se o ponto de corte seis ou mais condições médicas coexistentes (MM6+), como referido na metodologia. Através do modelo GEE não se verificou associação estatisticamente significativa de MM6+ com qualquer variável sociodemográfica.

As análises bi e multivariável da multimorbilidade foram realizadas para as variáveis sociodemográficas anteriormente descritas e para as de morbilidade, considerando-se estas as de maior relevância. Nesse sentido, para o ponto de corte (MM6+) como variável dependente analisou-se, através do modelo GEE, a sua associação com gravidade ou índice de Charlson, diagnósticos ou condições médicas e sistemas.

Os resultados da análise são sintetizados de forma tabelar, incluindo a frequência dos diagnósticos associados à multimorbilidade (MM6+), bem como a significância dessa associação (o valor de $p$ ) ou o Odds Ratio 
(OR) com o respetivo intervalo de confiança de 95\% (IC95 do OR).

A MM6+ (Quadro II) revelou associação estatisticamente significativa a nove condições médicas, sendo o OR superior para a hipertensão, seguindo-se a osteoartrose. A doença cerebrovascular e a hemiplegia tiveram $\mathrm{OR}$ acima de 7. O OR mais baixo, ainda assim de 3,183, verificou-se para a depressão. Para o ponto de corte MM6+ não se verificou associação estatisticamente significativa com demência.

Tendo subjacente que a complexidade da multimorbilidade pode estar associada ao envolvimento de diferentes aparelhos e sistemas

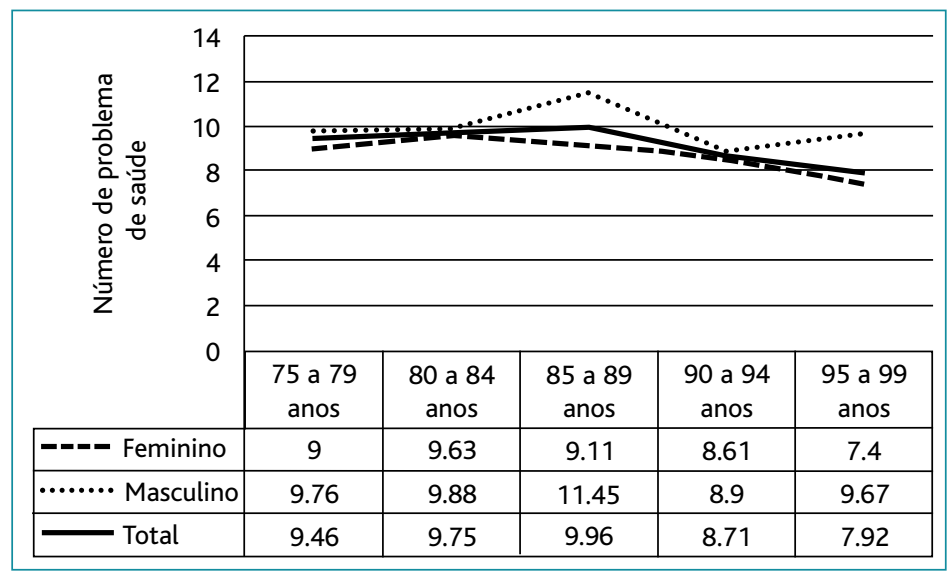

Figura 2. Distribuição do número médio de problemas por grupos etário e sexo. analisou-se a associação de MM6+ com cada sistema. Verificou-se o envolvimento de, pelo menos, três sistemas $(\mathrm{OR}=30,889)$ : circulatório $(\mathrm{OR}=11,258)$, músculo-esquelético $(\mathrm{OR}=5,646)$ e endócrino-metabólico (OR=2,925).

Quando se analisou a associação de MM6+ com gravidade de Charlson $(\geq 9)$ verificou-se uma associação estatisticamente significativa (OR=22,333; IC95\% 3,742133,299; $p=0,001$ ).

$\mathrm{Na}$ análise multivariável consideraram-se: as variáveis sociodemográficas (idade, sexo, escolaridade e isolamento), as 12 condições médicas mais frequentes e a hemiplegia que na análise bivariável esteve associada a MM6+, não incluída nos 12 diagnósticos mais frequentes. Nesta análise, das variáveis sociodemográficas apenas o sexo masculino esteve associado a MM6+ (OR=6,833; IC95\% 1,771-26,365; $p=0,005)$. Os problemas de saúde que na análise multivariável estiveram associados a MM6+ foram: doença cerebrovascular ( $\mathrm{OR}=25,136)$, osteoatrose (OR=24,698), hipertensão arterial (OR=18,709), depressão (OR=15,081), osteoporose $(\mathrm{OR}=9,492)$ e ansiedade $(\mathrm{OR}=4,482)$.

\section{Gravidade da multimorbilidade (índice de Charlson)}

O índice de Charlson permite introduzir a dimensão gravidade, pela ponderação específica de alguns dos problemas. Torna-se, pois, complementar da magnitude da multimorbilidade enquanto somatório do número de condições médicas por pessoa. O índice de Charlson nesta amostra de idosos (75 e mais anos) pode assumir valores entre 4 e 37, sabendo-se que o valor $\geq 5$ corresponde ao risco de morrer a três anos de $85 \%$.

O score médio do índice de Charlson foi de 8,5 (IC95\% 8,1-8,8) e o índice médio por grupo etário e sexo encontra-se na Figura 3.

Da observação da Figura 3 verifica-se que o índice de Charlson médio é superior no sexo masculino em todos os grupos etários, não sendo linear o seu aumento com a idade para os homens e registando-se um decréscimo com a idade para as mulheres. As curvas revelam comportamentos semelhantes às da média do número de problemas, distinguindo-se pelo acentuar da diferença entre sexos.

Com a finalidade de diferenciar os participantes de acordo com a gravidade estabeleceu-se como ponto de corte do índice de Charlson o valor 9, criando dois grupos quanto à gravidade: Grave (Charlson 4 a 9) e Muito grave (Charlson $\geq 9$ ). A análise da gravidade do Charlson fez-se para as variáveis sociodemográficas, tendo apenas o sexo revelado associação estatisticamente significativa $(p<0,001)$, parecendo ser o sexo feminino protetor (OR=0,456; IC95\% 0,331-0,629). Também para a magnitude da multimorbilidade se analisou, através do modelo GEE, a associação da gravidade de Charlson com: diagnósticos ou condições médicas, aparelhos ou sistemas e multimorbilidade. No Quadro III encontram-se, por ordem decrescente de OR, as condições médicas associadas a gravidade de multimorbilidade (índice de Charlson $\geq 9$ ).

A associação estatisticamente significativa da gravidade com as condições médicas que diferiram da magnitude da multimorbilidade corresponde a diagnósticos incluídos na escala de Charlson como: úlcera pép- 


\begin{tabular}{|c|c|c|c|}
\hline Condições médicas ou diagnósticos & $\mathrm{N}^{*}(\%)$ & OR(IC95\%) & $\mathbf{P}$ \\
\hline Hipertensão arterial (HTA) N=166 & $162 / 166(97,6 \%)$ & & \\
\hline Sem HTA (Ref) & $45 / 54(70,3 \%)$ & $15,219(5,295-43,742)$ & $<0,001$ \\
\hline Osteoartrose $\mathrm{N}=175$ & $168 / 175(96 \%)$ & $9,842(4,066-23,823)$ & \\
\hline Sem osteoartrose (Ref) & $39 / 55(79,9 \%)$ & & $<0,001$ \\
\hline Doença Cerebrovascular $\mathrm{N}=114$ & $111 / 114(97,4 \%)$ & & \\
\hline Sem doença cerebrovascular (Ref) & $96 / 116(82,8 \%)$ & $7,365(2,956-18,351)$ & $<0,001$ \\
\hline Diabetes $N=75$ & 73/75(97,3\%) & $6,231(1,559-24,901)$ & 0,010 \\
\hline Sem diabetes (Ref) & $134 / 155(86,5 \%)$ & & \\
\hline Insuficiência Cardíaca N= 79 & $77 / 79(97,5 \%)$ & & \\
\hline Sem insuficiência cardíaca (Ref) & $130 / 151(86,1 \%)$ & $6,066(1,881-19,559)$ & 0,003 \\
\hline Doença vascular periférica (DVP) N=100 & $96 / 100(96,0 \%)$ & & \\
\hline Sem DVP (Ref) & $111 / 130(85,4 \%)$ & $4,256(1,425-12,710)$ & 0,009 \\
\hline Perturbação do sono $\mathrm{N}=104$ & $99 / 104(95,2 \%)$ & $3,243(1,441-7,295)$ & 0,004 \\
\hline Sem perturbação do sono (Ref) & $108 / 126(85,7 \%)$ & & \\
\hline Ansiedade $\mathrm{N}=77$ & $74 / 77(96,1 \%)$ & $3,792(1,188-12,108)$ & 0,024 \\
\hline Sem ansiedade (Ref) & 133/153(86,9\%) & & \\
\hline Depressão $\mathrm{N}=90$ & $86 / 90(95,6 \%)$ & $3,183(1,208-8,386)$ & 0,019 \\
\hline Sem depressão (Ref) & $121 / 140(86,4 \%)$ & & \\
\hline
\end{tabular}

* MM6+ foi a variável dependente considerada. Em cada célula da coluna $\mathrm{N}$ encontra-se a negrito o número e a percentagem de idosos com a condição médica de entre os com seis ou mais condições médicas.

tica, coronariopatia, doença pulmonar obstrutiva crónica (DPOC) e tumor sólido não metastizado ou leucemia e linfoma. O diagnóstico não coincidente com os itens da escala de Charlson, embora previsto no questionário, e que surgiu associado a gravidade foi a obesidade. A hierarquia da associação foi diferente de MM6+.

Nesta análise das variáveis sociodemográficas apenas o sexo masculino esteve associado a gravidade de Charlson (OR=1,694; IC95\% 1,076-2,667; $p=0,023$ ).

A gravidade diferiu da medida de magnitude da multimorbilidade (MM6+) pela ausência de associação estatisticamente significativa com o aparelho músculo-esquelético. Na análise multivariável, os sistemas associados a gravidade de multimorbilidade foram: urológico ( $\mathrm{OR}=6,117)$, circulatório $(\mathrm{OR}=4,402)$, neurológico $(\mathrm{OR}=3,442)$, respiratório $(\mathrm{OR}=2,950)$, digestivo $(\mathrm{OR}=2,135)$ e endócrino-metabólico (OR=2,126).

Nesta amostra, a partir das seis condições médicas a associação do índice de Charlson muito grave e a mul- timorbilidade foi estatisticamente significativa, de MM6+ a MM8+, todavia com um OR decrescente: MM6+ $(\mathrm{OR}=22,333), \mathrm{MM} 7+(\mathrm{OR}=15,285)$ e MM8+ $(\mathrm{OR}=5,722)$.

\section{Padrão da multimorbilidade}

Na sequência da caracterização da magnitude da multimorbilidade (contagem de problemas) e da sua gravidade (índice de Charlson) efetuou-se a caracterização do padrão, isto é, perceber como se agregam os diagnósticos. Dos diagnósticos associados a uma média mais elevada de problemas por pessoa (Quadro IV) destacam-se a coronariopatia isquémica ( 11,64 condições por pessoa), a DPOC (11,38 condições por pessoa) e a insuficiência cardíaca ( 11,30 condições por pessoa).

Na caracterização do padrão de multimorbilidade através da análise multivariável de diagnósticos identificou-se uma diversidade de padrões de associação estatisticamente significativa desde diagnósticos associados a um, a dois e até oito condições médicas. As doenças vasculares foram as que revelaram associação 
estatisticamente significativa a maior número de diagnósticos (Quadro IV), nem todos do mesmo sistema.

As doenças osteoarticulares (osteoartrose e osteoporose) estiveram associadas apenas a um problema cada, apesar da sua elevada frequência na amostra, em particular a osteoartrose. Nesta análise, a osteoartrose, sendo o problema mais frequente, apenas esteve associada à obesidade e à osteoporose. A hipertensão arterial foi o diagnóstico mais frequentemente associado a outros de natureza vascular, metabólica ou osteoarticular. A insuficiência cardíaca foi o segundo diagnóstico associado a mais condições médicas de diferentes sistemas, sendo que duas (coronariopatia isquémica e doença cerebrovascular) têm nexo causal comum. Nesta amostra, a diabetes esteve apenas associada à hipertensão arterial e a demência à doença cerebrovascular e à úlcera crónica.

Os problemas neuropsiquiátricos tiveram duas combinações biunívocas que foram entre a depressão e a ansiedade e entre a demência e a doença cerebrovascular.

\section{DISCUSSÃO}

A população portuguesa revela uma tendência de envelhecimento demográfico, ${ }^{32-34}$ que os resultados do estudo parecem confirmar, como se pode inferir dos $71 \%$ da população ao cuidado das ECCI da RNCCI que cumpriam o critério de inclusão idade (75 e mais anos) (Figura 1). A média de idade dos participantes foi de 83,6 anos. Quanto ao sexo, as mulheres representam cerca de $54 \%$ dos participantes e são mais velhas (média de idade de 84,9 anos). Em suma, nesta amostra as mulheres estão mais representadas e são mais idosas, dados coerentes com o perfil do envelhecimento da população portuguesa. $^{23,35}$

A multimorbilidade encontra-se fortemente associada a determinantes sociais de saúde, sendo mais elevada em estratos sociais mais desfavorecidos. ${ }^{36}$ Quanto à caracterização sociodemográfica, um dos aspetos que importa realçar foi a baixa escolaridade dos participantes (cerca de 40,4\% dos idosos não tinha escolaridade e $48,3 \%$ tinha a escolaridade básica), superior aos resultados do estudo EPEPP (35\% de idosos com 75 anos e mais tinha menos de três anos de escolaridade). ${ }^{23}$ A educação é um forte preditor de resultados de saúde, ${ }^{37}$ isto é, em saúde indivíduos com mais educação be-

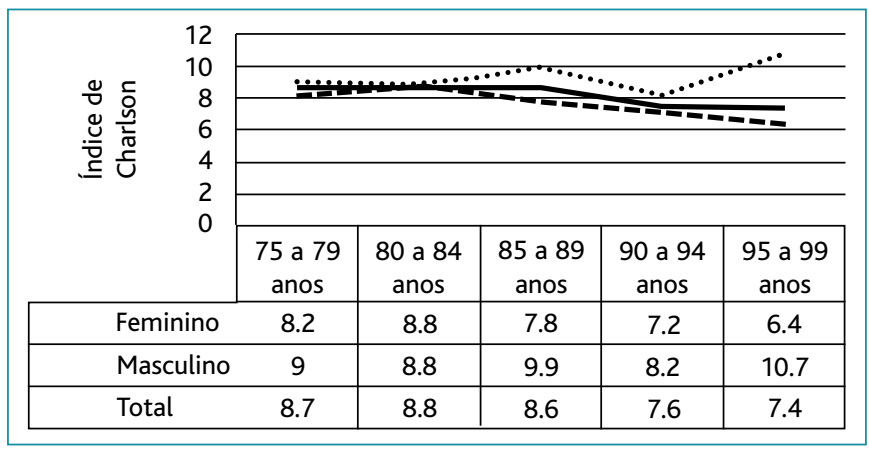

Figura 3. Distribuição do índice médio de Charlson por sexo e grupo etário.

neficiam de ambientes mais saudáveis, ${ }^{37}$ enquanto níveis de escolaridade mais baixos estão associados a baixa literacia e a piores resultados em saúde (e.g., taxa de mortalidade).$^{37-39}$ Se se utilizasse a educação como indicador económico ${ }^{40}$ poder-se-ia inferir ser uma população de baixo estrato social; no entanto, atendendo ao grupo etário e ao predomínio do sexo feminino, por razões de natureza sociocultural não se pode, com segurança, fazer essa afirmação.

O interesse crescente na multimorbilidade deve-se à sua associação com mortalidade, complexidade do cuidado, redução da qualidade vida ${ }^{3,7}$ e custos em saúde. ${ }^{41}$

Estudos realizados na população revelaram que a multimorbilidade está associada às idades mais avançadas, ${ }^{1-2,36,42-43}$ o que se confirma neste estudo, onde todos os idosos tinham pelo menos duas doenças crónicas, 207 dos 230 idosos (90\%) tinha seis ou mais diagnósticos por pessoa e apenas cerca de um quarto dos participantes não tinha oito ou mais condições médicas. A elevada frequência de multimorbilidade refletiu-se numa média de problemas por pessoa de 9,5 e num índice de Charlson 8,5, a par de um comportamento diferente entre sexos quanto à variabilidade do número médio de problemas por pessoa.

O comportamento das curvas da média de problemas por sexo mostrou, no masculino, um pico aos 85/89 anos e uma curva ascendente no grupo dos mais idosos, enquanto no sexo feminino se encontrou um esboço de um pico aos 80/84 anos, seguindo-se um declínio progressivo até à idade mais avançada. Confirmou-se, ainda, a associação estatisticamente significativa entre multimorbilidade e o sexo masculino $(\mathrm{OR}=2,452)$, ao contrário do verificado na literatura, ${ }^{43-44}$ embora semelhante ao estudo Português. ${ }^{6}$ 


\begin{tabular}{|c|c|c|c|}
\hline Condições médicas ou diagnósticos & $\mathbf{N}^{*}(\%)$ & OR(IC95\%) & $\mathbf{P}$ \\
\hline Coronariopatia $\mathrm{N}=58$ & $46 / 58(79,3 \%)$ & & \\
\hline Sem Coronariopatia (Ref) & $60 / 172(34,9 \%)$ & $7,055(2,710-18,371)$ & $<0,001$ \\
\hline Insuficiência cardíaca N= 79 & $58 / 79(73,4 \%)$ & & \\
\hline Sem insuficiência cardíaca (Ref) & $48 / 151(31,8 \%)$ & $5,926(2,991-11,740)$ & $<0,001$ \\
\hline Doença Cerebrovascular $\mathrm{N}=114$ & $76 / 114(66,7 \%)$ & & \\
\hline Sem doença cerebrovascular (Ref) & $30 / 116(25,9 \%)$ & $5,623(3,211-9,848)$ & $<0,001$ \\
\hline Hipertensão arterial (HTA) N=166 & $91 / 166(54,8 \%)$ & & \\
\hline Sem HTA (Ref) & $15 / 64(23,4 \%)$ & $4,255(2,246-8,058)$ & $<0,001$ \\
\hline Diabetes $\mathrm{N}=75$ & $51 / 75(68,0 \%)$ & & \\
\hline Sem diabetes (Ref) & $55 / 155(35,5 \%)$ & $3,802(2,071-6,979)$ & $<0,001$ \\
\hline Úlcera péptica N= 41 & $28 / 41(68,3 \%)$ & & \\
\hline Sem Úlcera péptica (Ref) & $78 / 189(41,3 \%)$ & $3,007(1,559-5,800)$ & 0,001 \\
\hline $\mathrm{DPOC} \mathrm{N}=34$ & $23 / 34(67,6 \%)$ & & \\
\hline Sem DPOC (Ref) & $83 / 196(42,3 \%)$ & $2,757(1,038-7,322)$ & 0,042 \\
\hline TSNMLL N= 40 & $26 / 40(65,0 \%)$ & & \\
\hline Sem TSNMLL (Ref) & $80 / 190(42,1 \%)$ & $2,481(1,212-5,078)$ & 0,013 \\
\hline Doença vascular periférica (DVP)N=100 & $58 / 100(58,0 \%)$ & & \\
\hline Sem DVP (Ref) & $48 / 130(36,9 \%)$ & $2,333(1,616-3,370)$ & $<0,001$ \\
\hline Arritimia cardíaca $\mathrm{N}=49$ & $29 / 49(59,2 \%)$ & & \\
\hline Sem arritimia cardíaca (Ref) & $77 / 181(42,5 \%)$ & $1,901(1,075-3,361)$ & 0,027 \\
\hline Obesidade $\mathrm{N}=45$ & $28 / 45(62,2 \%)$ & & \\
\hline Sem obesidade (Ref) & $78 / 185(42,2 \%)$ & $2,271(1,012-5,097)$ & 0,047 \\
\hline
\end{tabular}

* O índice de Charlson $\geq 9$ foi a variável dependente considerada. Em cada célula da coluna $\mathrm{N}$ encontra-se a negrito o número e a percentagem de idosos com a condição médica de entre os Índice de Charlson $\geq 9$

Quanto à magnitude da multimorbilidade, e porque nesta amostra abaixo de seis condições médicas por pessoa se verificou ausência de casos suficientes para cruzamento, analisou-se o ponto de corte: seis ou mais condições médicas coexistentes (MM6+). Através do modelo GEE, para a multimorbilidade MM6+ não se verificou associação estatisticamente significativa com qualquer variável sociodemográfica. Para o ponto de corte MM6+ não se verificou associação estatisticamente significativa com demência. A análise multivariável da associação entre MM6+ e os sistemas foi coerente com os dados da literatura, confirmando-se o envolvimento dos aparelhos circulatório (K), músculo-esquelético (L) e endócrino-metabólico ( $\mathrm{T}$ ) e com os agregados de patologias, clinicamente relevantes (e.g., aparelho circulatório e metabólico) $)^{10-11,14,45-46}$. Os resultados deste estudo podem, pois, contribuir para a caracterização da combinação entre múltiplas patologias e para a compreensão da complexidade da multimorbilidade. ${ }^{8,36}$

Outro dos aspetos que importa discutir é a gravidade da multimorbilidade que, apesar de não se encontrar claramente conceptualizada, ${ }^{15}$ tem utilizado como instrumentos de medida índices que agregam diagnósticos e os ponderam de acordo com o risco de morte, como o de Charlson. ${ }^{12,30-31}$ Analisou-se a associação entre a gravidade medida pelo índice de Charlson $(\geq 9)$ e a magnitude (número de problemas por pessoa), tendo-se verificado associação estatisticamente significativa; contudo, com tendência decrescente do OR entre MM6+ (OR=22,333) e MM8+ (OR=5,686). Desconhece-se o significado clínico do valor decrescente de OR, podendo ser explicado pelo facto de quando a magni- 


\begin{tabular}{|c|c|c|c|c|c|}
\hline & N. ${ }^{\circ}$ & Índice & & & \\
\hline $\begin{array}{l}\text { Diagnósticos mais frequentes } \\
\text { (variáveis dependentes) }\end{array}$ & $\begin{array}{l}\text { Problemas } \\
\text { Médio }\end{array}$ & $\begin{array}{l}\text { Charlson } \\
\text { Médio }\end{array}$ & $\begin{array}{l}\text { Problemas associados } \\
\text { (variáveis independentes) }\end{array}$ & OR(IC95\%) & $\mathbf{P}$ \\
\hline \multirow[t]{3}{*}{ Coronariopatia isquémica } & \multirow[t]{3}{*}{11,64} & \multirow[t]{3}{*}{10,50} & Insuficiência cardíaca & $4,433(2,165-9,078)$ & $<0,001$ \\
\hline & & & Hipertensão arterial & $4,118(1,624-10,445)$ & 0,003 \\
\hline & & & Cancro metastizado & $2,471(1,159-5,269)$ & 0,019 \\
\hline \multirow[t]{2}{*}{ Obesidade } & \multirow[t]{2}{*}{11,53} & \multirow[t]{2}{*}{9,27} & Insuficiência cardíaca & $4,317(1,781-10,464)$ & 0,001 \\
\hline & & & Hipertensão arterial & $4,139(1,867-9,173)$ & $<0,001$ \\
\hline DPOC & 11,38 & 9,56 & Insuficiência cardíaca & $2,711(1,082-6,794)$ & 0,033 \\
\hline \multirow[t]{7}{*}{ Insuficiência cardíaca } & \multirow[t]{7}{*}{11,30} & \multirow[t]{7}{*}{10,09} & Obesidade & $4,477(1,733-11,568)$ & 0,002 \\
\hline & & & Cancro metastizado & $4,318(1,991-9,364)$ & $<0,001$ \\
\hline & & & Arritmia cardíaca & $4,239(2,279-7,884)$ & $<0,001$ \\
\hline & & & Coronariopatia isquémica & $3,685(1,859-7,306)$ & $<0,001$ \\
\hline & & & Doença cerebrovascular & $2,424(1,420-4,137)$ & 0,001 \\
\hline & & & Osteoporose & $1,993(1,128-3,520)$ & 0,017 \\
\hline & & & Úlcera crónica de pele & $1,899(1,078-3,345)$ & 0,026 \\
\hline Diabetes & 10,99 & 10,03 & Hipertensão arterial & $3,020(1,826-4,994)$ & $<0,001$ \\
\hline \multirow[t]{2}{*}{ Arritmia cardíaca } & \multirow[t]{2}{*}{10,80} & \multirow[t]{2}{*}{9,06} & Insuficiência cardíaca & $4,601(2,421-8,744)$ & $<0,001$ \\
\hline & & & Hipertensão arterial & $2,691(1,243-5,827)$ & 0,012 \\
\hline \multirow[t]{3}{*}{ Doença vascular periférica } & \multirow[t]{3}{*}{10,70} & \multirow[t]{3}{*}{9,35} & Úlcera crónica de pele & $2,470(1,057-5,776)$ & 0,037 \\
\hline & & & Hipertensão arterial & $2,424(1,201-4,889)$ & 0,013 \\
\hline & & & Coronariopatia isquémica & $1,966(1,183-3,266)$ & 0,009 \\
\hline \multirow[t]{3}{*}{ Doença cerebrovascular } & \multirow[t]{3}{*}{10,58} & \multirow[t]{3}{*}{9,63} & Demência & $4,842(2,940-7,972)$ & $<0,001$ \\
\hline & & & Hipertensão arterial & $3,039(1,559-5,923)$ & 0,001 \\
\hline & & & Insuficiência cardíaca & $2,152(1,155-4,009)$ & 0,016 \\
\hline \multirow[t]{8}{*}{ Hipertensão arterial } & \multirow[t]{8}{*}{10,48} & \multirow[t]{8}{*}{9,07} & Obesidade & $5,306(2,567-10,966)$ & $<0,001$ \\
\hline & & & Coronariopatia isquémica & $4,056(1,622-10,139)$ & 0,003 \\
\hline & & & Diabetes & $3,535(1,558-8,021)$ & 0,003 \\
\hline & & & DPOC & $3,297(1,017-10,688)$ & 0,047 \\
\hline & & & Doença cerebrovascular & $3,275(1,729-6,206)$ & $<0,001$ \\
\hline & & & Arritmia cardíaca & $2,920(1,324-6,437)$ & 0,008 \\
\hline & & & Depressão & $2,621(1,261-5,449)$ & 0,010 \\
\hline & & & Doença vascular periférica & $2,311(1,178-4,531)$ & 0,015 \\
\hline \multirow[t]{4}{*}{ Ansiedade } & \multirow[t]{4}{*}{10,31} & \multirow[t]{4}{*}{8,31} & Depressão & $3,232(1,854-5,632)$ & $<0,001$ \\
\hline & & & Úlcera crónica de pele & $2,183(1,184-4,025)$ & 0,012 \\
\hline & & & Coronariopatia isquémica & $1,735(1,009-2,981)$ & 0,046 \\
\hline & & & Osteoporose & $1,714(1,037-2,830)$ & 0,035 \\
\hline
\end{tabular}


QUADRO IV. Problemas associados aos diagnósticos mais frequentes na análise multivariável (continuação)

\begin{tabular}{|c|c|c|c|c|c|}
\hline & N. ${ }^{\circ}$ & Índice & & & \\
\hline $\begin{array}{l}\text { Diagnósticos mais frequentes } \\
\text { (variáveis dependentes) }\end{array}$ & $\begin{array}{l}\text { Problemas } \\
\text { Médio }\end{array}$ & $\begin{array}{l}\text { Charlson } \\
\text { Médio }\end{array}$ & $\begin{array}{l}\text { Problemas associados } \\
\text { (variáveis independentes) }\end{array}$ & OR(IC95\%) & $\mathbf{P}$ \\
\hline \multirow[t]{2}{*}{ Depressão } & \multirow[t]{2}{*}{10,29} & \multirow[t]{2}{*}{8,41} & Ansiedade & $3,226(1,789-5,818)$ & $<0,001$ \\
\hline & & & Hipertensão arterial & $1,992(1,036-3,831)$ & 0,039 \\
\hline Dislipidémia & 10,20 & 8,63 & Diabetes & $2,129(1,170-3,876)$ & 0,013 \\
\hline Osteoartrose & 10,14 & 8,65 & Obesidade & $2,732(1,239-6,026)$ & 0,013 \\
\hline Osteoporose & 9,92 & 8,25 & Osteoatrose & $2,347(1,359-4,054)$ & 0,002 \\
\hline \multirow[t]{2}{*}{ Cancro metastizado } & \multirow[t]{2}{*}{9,49} & \multirow[t]{2}{*}{8,48} & Insuficiência cardíaca & $3,061(1,378-6,799)$ & 0,006 \\
\hline & & & Coronariopatia isquémica & $3,021(1,108-8,241$ & 0,031 \\
\hline \multirow[t]{3}{*}{ Demência } & \multirow[t]{3}{*}{9,47} & \multirow[t]{3}{*}{8,49} & Doença cerebrovascular & $4,514(2,917-6,988)$ & $<0,001$ \\
\hline & & & Úlcera crónica de pele & $2,725(1,435-5,177)$ & 0,002 \\
\hline & & & Osteoporose & $1,958(1,422-2,697)$ & $<0,001$ \\
\hline
\end{tabular}

tude da multimorbilidade aumenta podem ser contabilizados problemas com menor gravidade e não incluídos no índice de Charlson (e.g., osteoartrose, obesidade). Embora com a necessária precaução questiona-se se o ponto de corte MM6+ não poderá ser uma medida simples que integra magnitude e gravidade de multimorbilidade e se não poderá vir a ser uma possível alternativa à definição de multimorbilidade corrente (coexistência de duas ou mais doenças crónicas), que parece inadequada às pessoas com mais idade por representar a norma. ${ }^{5-6,47}$ Outro dado a reforçar esta questão foi o facto de se ter verificado associação estatisticamente significativa entre MM6+ e morbilidade multissistémica (envolvimento de três ou mais sistemas, MS3+). Tendo em conta a definição de multimorbilidade complexa ${ }^{3}$ definiu-se como multimorbilidade multissistémica aquela que afeta três ou mais aparelhos ou sistemas, ${ }^{48}$ a qual revelou associação estatisticamente significativa com gravidade através do índice de Charlson (MS3+; OR=3,171). Todavia, quanto a esta inferência sobre gravidade, conferida por seis ou mais doenças crónicas (MM6+) ou três ou mais sistemas (MS3+) envolvidos, serão necessários outros estudos que incluam outras medidas de gravidade clínica, como a natureza das condições médicas associadas e o estadio da doença (e.g., grau de insuficiência cardíaca).

Conhecer o padrão de multimorbilidade é uma ne- cessidade crescente, nomeadamente, a complexidade dos agregados de doenças crónicas e suas combinações, ${ }^{8-9,14}$ pelo seu impacto na gestão da doença, na integração de cuidados, nos cuidados centrados no paciente e na melhoria da qualidade de vida. ${ }^{8-9,45}$ No sentido de conhecer o padrão de multimorbilidade socorreu-se da análise multivariável através do modelo GEE. Nesta análise, a osteoartrose, sendo o problema mais prevalente, apenas esteve associada à obesidade. A hipertensão arterial foi o diagnóstico associado a mais condições médicas de natureza diversa: vascular, metabólica ou de saúde mental. A insuficiência cardíaca foi o segundo diagnóstico associado a mais condições médicas de diferentes aparelhos ou sistemas, sendo que duas (coronariopatia isquémica e doença cerebrovascular) são consideradas com nexo fisiopatológico. Curiosamente, nesta amostra a diabetes esteve apenas associada à hipertensão arterial. A demência esteve associada à doença cerebrovascular e à úlcera crónica. As associações identificadas através do modelo GEE revelaram-se estatística e clinicamente relevantes (Quadro IV). Observando as combinações de risco destacam-se associações que conferem coerência clínica aos resultados, pela associação causal, como as osteoarticulares entre si (osteoporose, osteoartrose), a osteoartrose e a obesidade, a demência e a doença cerebrovascular ou a insuficiência cardíaca e a úlcera crónica de pele. Ques- 
tiona-se se não podem os modelos lineares generalizados ser instrumentos de análise que permitem identificar agregados pela frequência dos problemas na população (não causal), como hipertensão e osteoartrose, e associações fisiopatológicas (causais), como insuficiência cardíaca e coronariopatia?

As limitações do estudo decorrem da metodologia escolhida (desenho e amostragem), a qual teve subjacentes princípios de exequibilidade, gestão limitada de recursos humanos, financeiros e temporais. A inclusão de idosos com 75 anos e mais anos estreitou a possibilidade de análise comparativa da multimorbilidade com grupos mais novos (e.g., 65 a 74 anos), em que a idade pode ser um fator de confundimento.

Outro dos aspetos metodológicos discutíveis foi a utilização de diagnósticos autorreportados, ${ }^{49}$ não medicamente confirmados. Contudo, tentou minimizar-se este constrangimento complementando a informação diagnóstica, pela investigadora médica, através da análise da terapêutica em curso e da consulta da informação clínica disponível (e.g., notas de alta hospitalar ou relatórios clínicos), à semelhança de outros estudos de multimorbilidade. ${ }^{47,50}$

A magnitude da multimorbilidade (contagem de condições médicas) é uma limitação deste estudo e de outros de multimorbilidade por usar a soma de doenças como escala ordinal, sem considerar a gravidade ou o estadio da doença. ${ }^{51}$

O índice de Charlson inclui a gravidade da doença para um número limitado de diagnósticos e omite a doença mental que, em contexto de cuidados de saúde primários, confere substancial morbilidade.$^{58}$ Não se pode com o índice Charlson, tal como com outros índices, medir com precisão a carga de multimorbilidade devido à variação na sensibilidade e especificidade dos diferentes diagnósticos, à falência na inclusão da gravidade e duração da doença, as quais podem estar relacionadas com o resultado. ${ }^{52}$

Outro dos aspetos questionáveis é ter considerado como entidades nosológicas diferentes condições médicas com nexo causal comum, como a doença cerebrovascular e a hemiplegia (sequela de acidente vascular cerebral) ou ansiedade e perturbação do sono (sintomas diferentes da mesma perturbação). ${ }^{3}$ Contudo, a categorização como entidades distintas tem relevância numa perspetiva centrada no paciente, em que a doen- ça e a sua vivência podem ser entidades diferentes..$^{53-55}$

Consideram-se pontos fortes os que tornam única esta investigação, como o número de visitas domiciliárias com uma distribuição sistematizada que torna a amostra de idosos dependentes a receber cuidados de saúde domiciliários representativa da região de Lisboa eVale do Tejo. Os resultados permitiram obter conhecimento sobre a magnitude, gravidade e padrão da multimorbilidade, o que poderá ser útil para a reflexão sobre o modelo organizativo de cuidados da RNCCI na sua vertente domiciliária (ECCI) e consequente alocação de recursos.

Uma vez que os doentes inseridos em ECCI são uma população geograficamente dispersa e dinâmica que impossibilitava a aleatorização dos participantes justificou o processo de amostragem por clusters, ${ }^{56-57}$ à semelhança de outros estudos epidemiológicos, ${ }^{25-29}$ o que permitiu a análise dos dados através do modelo de linear generalizado por equações de estimação generalizada (GEE) com estrutura de correlação uniforme dentro de cada cluster (ECCI).

A colheita de dados por entrevista e aplicação do mesmo questionário a todos os participantes pelo investigador facilitou a obtenção de informação padronizada, não alcançável através de bases de dados, acrescendo o consentimento informado livre e esclarecido prévio dos participantes.

Estudou-se a multimorbilidade através das suas diversas dimensões: magnitude (contagem), gravidade (índice de Charlson) e padrão (agregados ou combinações).

A análise multivariável da associação entre MM6+e os aparelhos e sistemas foi coerente com os dados da literatura, confirmando-se o envolvimento dos aparelhos circulatório (K), músculo-esquelético (L) e endócrino-metabólico ( $\mathrm{T}$ ) e com os agregados de patologias, clinicamente relevantes (e.g., aparelho circulatório e metabólico). ${ }^{10-11,14,45-46}$ Os resultados deste estudo podem, pois, contribuir para a caracterização da combinação entre múltiplas patologias e para a compreensão da complexidade da multimorbilidade. ${ }^{8,36}$

Os resultados deste estudo revelam validade interna conferida pela coerência clínica, como as combinações com relação fisiopatológica como a osteoartrose e obesidade, a insuficiência cardíaca e a coronariopatia isquémica ou a demência e a doença cerebrovascular.

Realçam-se os seguintes aspetos como revelando va- 
lidade externa e conferindo consistência aos resultados:

- Coerência sociodemográfica: A maior representatividade das mulheres (54\%) que são mais velhas (média de idade 84,9 anos), dados coerentes com os do envelhecimento da população portuguesa. ${ }^{23,35} \mathrm{~A}$ distribuição geográfica dos participantes, sendo a região da Grande Lisboa a mais representada (53,5\%), em concordância com os dados do Censo de 2011 para a região de Lisboa e Vale do Tejo. ${ }^{24,32-34}$

- Estudos realizados na população revelaram que a multimorbilidade estava associada a idades mais avançadas, ${ }^{1-2,36,42}$ o que se confirma neste estudo, onde todos os idosos tinham pelo menos duas doenças crónicas e $90 \%$ tinha seis ou mais, o que se refletiu numa média de problemas por pessoa de 9,5 e num índice de Charlson 8,5. A prevalência de multimorbilidade foi, assim, semelhante à de estudos que não limitaram o número de condições médicas crónicas consideradas. ${ }^{1,3}$

Em síntese, os resultados revelaram validade interna e externa, o que confere robustez ao estudo apesar das limitações anteriormente identificadas.

Tem surgido um interesse crescente na multimorbilidade pela sua associação com a mortalidade, complexidade do cuidado, redução da qualidade de vida ${ }^{3,7}$ e custos em saúde..$^{13,41}$ Numa altura em que vários países estão a proceder a grandes reformas dos cuidados de saúde primários, incluindo Portugal, a multimorbilidade parece ser um motor de mudança, uma vez que se reflete numa mudança do paradigma da gestão de serviços de saúde, centrada em doença única, para uma visão mais holística dos pacientes e uma «abordagem generalista» dos cuidados. ${ }^{7}$ Fica, em perspetiva, a necessidade de mais investigação, nomeadamente em modelos de cuidados e políticas de saúde.

\section{REFERÊNCIAS BIBLIOGRÁFICAS}

1. Zellweger U, Bopp M, Holzer BM, Djalali S, Kaplan V. Prevalence of chronic medical conditions in Switzerland: exploring estimates validity by comparing complementary data sources. BMC Public Health. 2014;14:1157.

2. Haregu T, Oldenburg B, Setswe G, Elliott J. Perspectives, constructs and methods in the measurement of multimorbidity and comorbidity: a critical review. Internet J Epidemiol. 2012;10(2):1-9.

3. Harrison C, Britt H, Miller G, Henderson J. Examining different measures of multimorbidity, using a large prospective cross-sectional study in Australian general practice. BMJ Open. 2014;4(7):e004694.

4. Diederichs C, Berger K, Bartels DB. The measurement of multiple chronic diseases: a systematic review on existing multimorbidity indices. J Gerontol A Biol Sci Med Sci. 2011;66A(3):301-11.
5. Violan C, Foguet-Boreu Q, Flores-Mateo G, Salisbury C, Blom J, Freitag M, et al. Prevalence, determinants and patterns of multimorbidity in primary care: a systematic review of observational studies. PLoS One. 2014;9(7):e102149.

6. Prazeres F, Santiago L. Prevalence of multimorbidity in the adult population attending primary care in Portugal: a cross-sectional study. BMJ Open. 2015;5(9):e009287.

7. Fortin M, Stewart M, Poitras ME, Almirall J, Maddocks H. A systematic review of prevalence studies on multimorbidity: toward a more uniform methodology. Ann Fam Med. 2012;10(2):142-51.

8. Islam MM, Valderas JM, Yen L, Dawda P, Jowsey T, McRae IS. Multimorbidity and comorbidity of chronic diseases among the senior Australians: prevalence and patterns. PLoS One. 2014;9(1):e83783.

9. Taylor AW, Price K, Gill TK, Adams R, Pilkington R, Carrangis N, et al. Multimorbidity - not just an older person's issue: results from an Australian biomedical study. BMC Public Health. 2010;10:718.

10. Prados-Torres A, Calderón-Larrañaga A, Hancco-Saavedra J, Poblador-Plou $B$, van den Akker M. Multimorbidity patterns: a systematic review. J Clin Epidemiol. 2014;67(3):254-66.

11. Olaya B, Moneta MV, Caballero FF, Tyrovolas S, Bayes I, Ayuso-Mateos JL, et al. Latent class analysis of multimorbidity patterns and associated outcomes in Spanish older adults: a prospective cohort study. BMC Geriatr. 2017;17(1):186.

12. De Groot V, Beckerman H, Lankhorst GJ, Bouter LM. How to measure comorbidity: a critical review of available methods. J Clin Epidemiol. 2003;56(3):221-9.

13. Huntley AL, Johnson R, Purdy S, Valderas JM, Salisbury C. Measures of multimorbidity and morbidity burden for use in primary care and community settings: a systematic review and guide. Ann Fam Med. 2012;10(2):134-41.

14. Schäfer I, von Leitner EC, Schön G, Koller D, Hansen H, Kolonko T, et al. Multimorbidity patterns in the elderly: a new approach of disease clustering identifies complex interrelations between chronic conditions. PLoS One. 2010;5(12):e15941.

15. Valderas JM, Starfield B, Sibbald B, Salisbury C, Roland M. Defining comorbidity: implications for understanding health and health services. Ann Fam Med. 2009;7(4):357-63.

16. Deyo RA, Cherkin DC, Ciol MA. Adapting a clinical comorbidity index for use with ICD-9-CM administrative databases. J Clin Epidemiol. 1992;45(6):613-9.

17. Boeckxstaens P, Vaes B, Legrand D, Dalleur O, De Sutter A, Degryse JM. The relationship of multimorbidity with disability and frailty in the oldest patients: a cross-sectional analysis of three measures of multimorbidity in the BELFRAIL cohort. Eur J Gen Pract. 2015;21(1):39-44.

18. Gomes B, Sarmento VP, Ferreira PL, Higginson IJ. Estudo epidemiológico dos locais de morte em Portugal em 2010 e comparação com as preferências da população Portuguesa [Epidemiological study of place of death in Portugal in 2010 and comparison with the preferences of the Portuguese population]. Acta Med Port. 2013;26(4):327-34. Portuguese

19. Decreto-Lei $n^{\circ} 101 / 2006$, de 6 de junho. Diário da República. $1^{\text {a }}$ Série$A(106): 3856-65$

20. Unidade de Missão para os Cuidados Continuados. Cuidados continuados integrados nos cuidados de saúde primários: carteira de serviços [Internet]. Lisboa: Ministério da Saúde; 2007. Available from: http://www2.acss.minsaude.pt/Portals/0/Cuidados\%20Continuados\%20nos\%20CSP.pdf

21. Lopes M, Mendes F, Escoval A, Agostinho M, Vieira C, Vieira I, et al. Cuidados continuados integrados: analisando o presente, perspectivando o futuro. In: Direção-Geral da Saúde. Plano nacional de saúde 2011-2016. Lisboa: DGS; 2010. p. 65-76.

22. Rede Nacional de Cuidados Continuados Integrados. Manual do prestador: recomendacoes para a melhoria continua [Internet]. Lisboa: Unidade de Missão dos Cuidados Continuados Integrados; 2011. Available from: http://www.acss.min-saude.pt/wp-content/uploads/2016/10/Man_ Prestador_UMCCI-RNCCI.pdf 
23. Oliveira CR, Rosa MS, Pinto AM, Botelho MA, Morais A, Verissimo MT. Estudo do perfil do envelhecimento da população Portuguesa. Coimbra: Faculdade de Medicina da Universidade de Coimbra; 2010. ISBN 9789898445001

24. Tavares A, Coelho MA, Rascôa CL. Perfil de Saúde 2015 e seus determinantes da Região de Lisboa e Vale do Tejo (Vol. 1). Lisboa: Administração Regional de Saúde de Lisboa e Vale do Tejo; 2015.

25. Carlin JB, Hocking J. Design of cross-sectional surveys using cluster sampling: an overview with Australian case studies. Aust N Z J Public Health. 1999;23(5):546-51.

26. Scheffers-Barnhoorn MN, van Haastregt JC, Schols JM, Kempen GI, van Balen R, Visschedijk JH, et al. A multi-component cognitive behavioural intervention for the treatment of fear of falling after hip fracture (FIT-HIP): protocol of a randomised controlled trial. BMC Geriatr. 2017;17(1):71.

27. Zapata-Ossa HJ, Cubides-Munévar AM, López MC, Pinzón-Gómez EM, Filigrana-Villegas PA, Cassiani-Miranda CA. Muestreo por conglomerados en encuestas poblacionales [Cluster sampling in health surveys]. Rev Salud Publica. 2010;13(1):141-51. Spanish

28. Masood M, Reidpath DD. Intraclass correlation and design effect in BMI, physical activity and diet: a cross-sectional study of 56 countries. BMJ Open. 2016;6(1):e008173.

29. Bell BA, Onwuegbuzie AJ, Ferron JM, Jiao QG, Hibbard ST, Kromrey JD. Use of design effects and sample weights in complex health survey data: a review of published articles using data from 3 commonly used adolescent health surveys. Am J Public Health. 2012;102(7):1399-405.

30. Charlson M, Szatrowski TP, Peterson J, Gold J.Validation of a combined comorbidity index. J Clin Epidemiol. 1994;47(11):1245-51.

31. Quan H, Li B, Couris CM, Fushimi K, Graham P, Hider P, et al. Updating and validating the charlson comorbidity index and score for risk adjustment in hospital discharge abstracts using data from 6 countries. Am J Epidemiol. 2011;173(6):676-82.

32. Instituto Nacional de Estatística. 25 de Abril: 40 anos de estatísticas. Lisboa: INE; 2014. ISBN 9789892502885

33. Instituto Nacional de Estatística. Estatísticas demográficas 2013. Lisboa: INE; 2014. ISBN 9789892502731

34. Instituto Nacional de Estatística. Indicadores sociais 2011. Lisboa: INE; 2012. ISBN 9789892501758

35. Delgado A, Wall K, editors. Familias nos Censos 2011: diversidade e mudança. Lisboa: Instituto Nacional de Estatística; Instituto de Ciências Sociais; 2014. ISBN 9789892502908

36. St John PD, Tyas SL, Menec V, Tate R Multimorbidity, disability, and mortality in community-dwelling older adults. Can Fam Physician. 2014;60(5): e272-80.

37. Zimmerman E, Woolf SH. Understanding the relationship between education and health [Internet]. Washington: Institute of Medicine of the National Academies; 2014. Available from: https://nam.edu/wp-content/ uploads/2015/06/BPH-UnderstandingTheRelationship1.pdf

38. Pedro AR, Amaral $O$, Escoval A. Literacia em saúde, dos dados à ação: tradução, validação e aplicação do European Health Literacy Survey em Portugal [Health Literacy, from data to action: translation, validation and application of the European Health Literacy Survey in Portugal]. Rev Port Saúde Pública. 2016;34(3):259-75. Portuguese

39. Annarumma C, Palumbo R. Contextualizing health literacy to health care organizations: exploratory insights. J Health Manag. 2016;18(4): 611-24.

40. OECD. Education at a glance 2016: OECD indicators. Paris: OECD; 2016. ISBN 9789264259805

41. Salive ME. Multimorbidity in older adults. Epidemiol Rev. 2013;35:75-83.

42. Marventano S, Ayala A, Gonzalez N, Rodríguez-Blázquez C, Garcia-Gutier- rez S, Forjaz MJ. Multimorbidity and functional status in institutionalized older adults. Eur Geriatr Med. 2016;7(1):34-9.

43. Rizza A, Kaplan V, Senn O, Rosemann T, Bhend H, Tandjung R. Age- and gender-related prevalence of multimorbidity in primary care: the Swiss FIRE project. BMC Fam Pract. 2012;13:113.

44. Abad-Díez JM, Calderón-Larrañaga A, Poncel-Falcó A, Poblador-Plou B, Calderón-Meza JM, Sicras-Mainar A, et al. Age and gender differences in the prevalence and patterns of multimorbidity in the older population. BMC Geriatr. 2014;14:75.

45. Foguet-Boreu Q, Violán C, Rodriguez-Blanco T, Roso-Llorach A, Pons-Vigués M, Pujol-Ribera E, et al. Multimorbidity patterns in elderly primary health care patients in a South Mediterranean European region: a cluster analysis. PLoS One. 2015;10(11):e0141155.

46. Kirchberger I, Meisinger C, Heier M, Zimmermann AK, Thorand B, Autenrieth $\mathrm{CS}$, et al. Patterns of multimorbidity in the aged population: results from the KORA-Age study. PLoS One. 2012;7(1):e30556.

47. Mokraoui NM, Haggerty J, Almirall J, Fortin M. Prevalence of self-reported multimorbidity in the general population and in primary care practices: a cross-sectional study. BMC Res Notes. 2016;9:314.

48. Britt HC, Harrison CM, Miller GC, Knox SA. Prevalence and patterns of multimorbidity in Australia. Med J Aust. 2008;189(2):72-7.

49. Gross R, Bentur N, Elhayany A, Sherf M, Epstein L. The validity of self-reports on chronic disease: characteristics of underreporters and implications for the planning of services. Public Health Rev. 1996;24(2):167-82.

50. Harrison C, Henderson J, Miller G, Britt $H$. The prevalence of diagnosed chronic conditions and multimorbidity in Australia: a method for estimating population prevalence from general practice patient encounter data. PLoS One. 2017;12(3):e0172935.

51. Marengoni A, Angleman S, Fratiglioni L. Prevalence of disability according to multimorbidity and disease clustering: a population-based study. J Comorb. 2011;1:11-8.

52. Ording AG, Sørensen HT. Concepts of comorbidities, multiple morbidities, complications, and their clinical epidemiologic analogs. Clin Epidemiol. 2013;5:199-203.

53. McWhinney IR. The importance of being different. $\mathrm{Br} J$ Gen Pract. 1996;46:433-6.

54. McWhinney IR. Illness, suffering and healing. In: McWhinney IR. A textbook of family medicine. 2nd ed. New York: Oxford University Press; 1997. p. 83103. ISBN 9780195115185

55. Stewart M. Towards a global definition of patient centred care. BMJ. 2001;322(7284):444-5.

56. Rutterford C, Copas A, Eldridge S. Methods for sample size determination in cluster randomized trials. Int J Epidemiol. 2015;44(3):1051-67.

57. Van Breukelen GJ, Candel MJ. Calculating sample sizes for cluster randomized trials: we can keep it simple and efficient! J Clin Epidemiol. 2012;65(11):1212-8.

\section{CONFLITO DE INTERESSES}

Os autores declaram não ter conflitos de interesses.

\section{ENDEREÇO PARA CORRESPONDÊNCIA}

Paula Broeiro-Gonçalves

E-mail: paulabroeirorpmgf@gmail.com

https://orcid.org/0000-0002-5013-6171

Recebido em 05-05-2018

Aceite para publicação em 24-01-2019 


\section{ABSTRACT}

\section{MAGNITUDE, PATTERN AND SEVERITY OF MULTIMORBIDITY IN ELDERLY PEOPLE FOLLOWED BY INTEGRATED CONTINUING CARE TEAMS: A CROSS-SECTIONAL STUDY}

Objective: To characterise the magnitude, severity and pattern of the multimorbidity. To verify which association exists between multimorbidity dimensions and medical conditions or systems.

Study type: Cross-sectional study, with descriptive and analytical component, by interview.

Setting: Integrated Continuing Care teams (ECCl) of Lisbon and Tagus Valley region.

Participants: Elderly aged 75 years and over.

Methods: Sample size ( $n=114$ elderly) was calculated for a $5 \%$ error margin and a $95 \%$ confidence interval, corrected for the design effect ( $n=228$ elderly). Sampling was performed using randomised selected clusters (ECCI). The analysis was performed through the generalised linear model - GEE using the IBM SPSS version 24.0 tool for the MAC OS operating system.

Results: 230 elderly participated, 54\% female, mean age 83.6 years, low schooling ( $40 \%$ without education), and $14.8 \%$ living alone. The total number of different medical conditions reported was 121. The average number of problems per person was 9.5 , and the average Charlson index was 8.5. There was an association between male gender and both magnitude $(\mathrm{OR}=2.452)$ and severity $(O R=22.333)$ of multimorbidity. Severity was associated with: multimorbidity defined as six or more conditions $(O R=22.333)$, and three or more systems $(O R=3.171)$. Ischemic coronary disease was associated with the highest mean number of conditions (11.64), and with Charlson's index (10.50). Hypertension and heart failure were the diagnoses with the most associated problems and more than three systems.

Conclusions: The population followed-up in ECCI is elderly and presents a high multimorbidity. A difference between genders was confirmed, with women older and men presenting with higher and more severe multimorbidity. The definition of multimorbidity of six or more problems was associated with multisystemic disease and severity.

Keywords: Multimorbidity; Comorbidity; Multimorbidity pattern; Elderly; Home visit. 